\title{
El registro fósil de plesiosaurios (Diapsida, Sauropterygia) en el Perú
}

\author{
The fossil register of plesiosaurs (Diapsida, Sauropterygia) in Peru
}

\section{Ivan Meza-Velez *1}

https://orcid.org/0000-0002-6217-8002

imv2999@gmail.com

\section{José O'Gorman 2}

https://orcid.org/0000-0001-9279-6314

joseogorman@fcnym.unlp.edu.ar

\section{*Corresponding author}

1 Departamento de Paleontología de Vertebrados del Museo de Historia Natural - Universidad Nacional Mayor de San Marcos.

2 División Paleontología Vertebrados, Museo de La Plata, Universidad Nacional de La Plata, Paseo del Bosque s/n., B1900FWA, La Plata, Argentina-CONICET: Consejo Nacional de InvestigacionesCientíficas y Técnicas, Argentina.

\section{Citación}

Meza-Velez I, O'Gorman J. 2021. El registro fósil de plesiosaurios (Diapsida, Sauropterygia) en el Perú. Revista peruana de biología 28(2): e20467 (Mayo 2021). doi: http://dx.doi. org/10.15381/rpb.v28i2.20467

$\begin{array}{ll}\text { Presentado: } & \text { 29/04/2020 } \\ \text { Aceptado: } & \text { 26/02/2021 } \\ \text { Publicado online: } & \text { 25/05/2021 } \\ & \\ \text { Editor: } & \text { Cesar Aguilar }\end{array}$

\section{Resumen}

Se realiza un recuento de los registros de plesiosaurios de Perú y se describe material referido a plesiosauria depositado en el Museo de Historia Natural de la UNMSM.El registro de plesiosaurios de Perú se extiende entre el ValanginianoHauteriviano (Cretácico Inferior) hasta el Maastrictiano (Cretácico Superior). En relación con la posición taxonómica, el material es referible a Plesiosauria indet., Elasmosauridae indet., mientras que el nuevo material estudiado es posiblemente referible a Pliosauroidea indet., constituyendo el primer registro de este grupo en el Perú. Asimismo, el plesiosaurio del Morro Solar es el único del Cretácico Inferior y el más antiguo de Perú.

\section{Abstract}

In this paper, the plesiosaur records for Peru are reviewed and material referring to plesiosauria deposited in the Natural History Museum of the UNMSM is described. The plesiosaur records of Peru are extended since the Valanginian-Hauterivian (Lower Cretaceous) to the Maastrictian (Upper Cretaceous). In relation to the taxonomic position, the material is attributable to Plesiosauria indet., Elasmosauridae indet., while the new material studied is possibly Pliosauroidea indet., and will be the first records of this group in Peru. Likewise, the Morro Solar plesiosaur is the only one from the Lower Cretaceous and the oldest in Peru.

Palabras clave:

Plesiosauria; Cretácico; Rentema; Morro Solar; Paita.

Keywords:

Plesiosauria; Cretaceous; Rentema; Morro Solar; Paita.

\section{Introducción}

Los plesiosaurios son un orden de reptiles marinos cuyo biocrón se extiende desde el Triásico Temprano hasta el límite K/Pg (Rieppel 1997, Rieppel 2000, Wintrich et al. 2017, O'Gorman et al. 2019). Entre las modificaciones del plan corporal básico de un tetrápodo se destaca la modificación de las extremidades en aletas con una marcada hiperfalangia (Romer 1966, O'Keefe 2001, Benson \& Druckenmiller 2014). El registro fósil de este grupo es cosmopolita, habiéndose registrado en todos los continentes, incluyendo la Antártida (Welles 1952, 1962, Brown 1981, Carpenter 1999, 0'Keefe 2004, O'Gorman 2012, Otero et al. 2014; Bardet et al. 2014; Bensony Druckenmiller 2014, Kear et al. 2018). Dentro del clado Plesiosauria, se distinguen dos morfotipos basados en sus proporciones corporales: plesiosauriomorfo y pliosauriomorfo (Welles 1943, O'Keefe 2002), los primeros poseen cuello largo y cabeza pequeña y los segundos cuello corto y cabeza alargada y grande. 
En Sudamérica, los plesiosaurios han sido registrados en Colombia (Welles 1962, Hampe 2005, ParamoFonseca et al. 2015), Venezuela (Colbert 1949), Perú (Jaillard et al. 1985, Meza-Velez \& O'Gorman 2020), Chile (Otero et al. 2015), Brasil (Woodward 1891, Vilas-Bôas \& Carvalhos 2001), Argentina (Gasparini \& Spalletti 1990, Gasparini \& De La Fuente 2000, Gasparini \& O'Gorman 2014, O'Gorman 2016).

Particularmente, en las costas del Pacífico de Sudamérica solo hay tres registros del Cretácico Inferior: el elasmosáurido Callawayasaurus colombiensis (Welles 1962), hallado en Colombia, restos referidos a Plesiosauria indet. provenientes de Perú (Meza-Vélez \& O'Gorman 2020), y finalmente el registro de elasmosáuridos indeterminados de la Formación Agrio, Argentina (Lazo \& Cichowolski 2003, O'Gorman et al. 2015a).

El registro de plesiosaurios del Perú se limita a cuatro (Fig. 1) caracterizados por materiales fragmentarios y falta de material diagnóstico. El primer reporte de reptiles marinos en Perú es mencionado en Taipe et al. (2004), referido al registro geológico mencionado en un reporte geológico para la International Petroleum Co., Ltd (Fischer 1956), donde se menciona la presencia de vertebrados en la península de Paita, y que años después fueron identificados por Natalie Bardet (Museo Nacional de París) como una mandíbula de plesiosaurio proveniente de la Formación Cenizo, Cretácico Superior,
Maastrichtiano, Playa Perico, península de Paita, además de fragmentos de cráneo de mosasaurio.

El primer registro de un plesiosaurio en Perú fue dado a conocer por Jaillard et al. (1985). En este trabajo, y en el marco de un análisis estratigráfico de la región de Jaén, se registraron cinco vértebras cervicales de un plesiosaurio hallado al norte del pueblo de Rentema, en el Pongo de Rentema. Una de las vértebras fue llevada a París y cuatro fueron llevadas al Museo Javier Prado de Lima, hoy Museo de Historia Natural de la Universidad Nacional Mayor de San Marcos (Jaillard et al. 1985). Sin embargo, no se registró en la mencionada contribución el número de colección para las mencionadas vértebras. Durante la búsqueda en el Departamento de Paleontología de Vertebrados del Museo de Historia Natural se encontraron dos vértebras de plesiosaurio (MUSM 3894), cuyos registros en la base de datos coinciden con los datos mencionados por Jaillard et al. (1985), concluyendo que es muy probable sean parte de los materiales depositados originalmente en el Museo Javier Prado de Lima (ver Discusión).

Un tercer hallazgo fue mencionado en Poiré et al. (2016), se trata de un hueso que podría pertenecer a un reptil marino (cf. Plesiosauria). El último registro de un plesiosaurio en Perú es el hallado en el Morro Solar de Lima (Meza-Vélez \& O'Gorman 2020) proveniente de niveles del Cretácico Inferior.
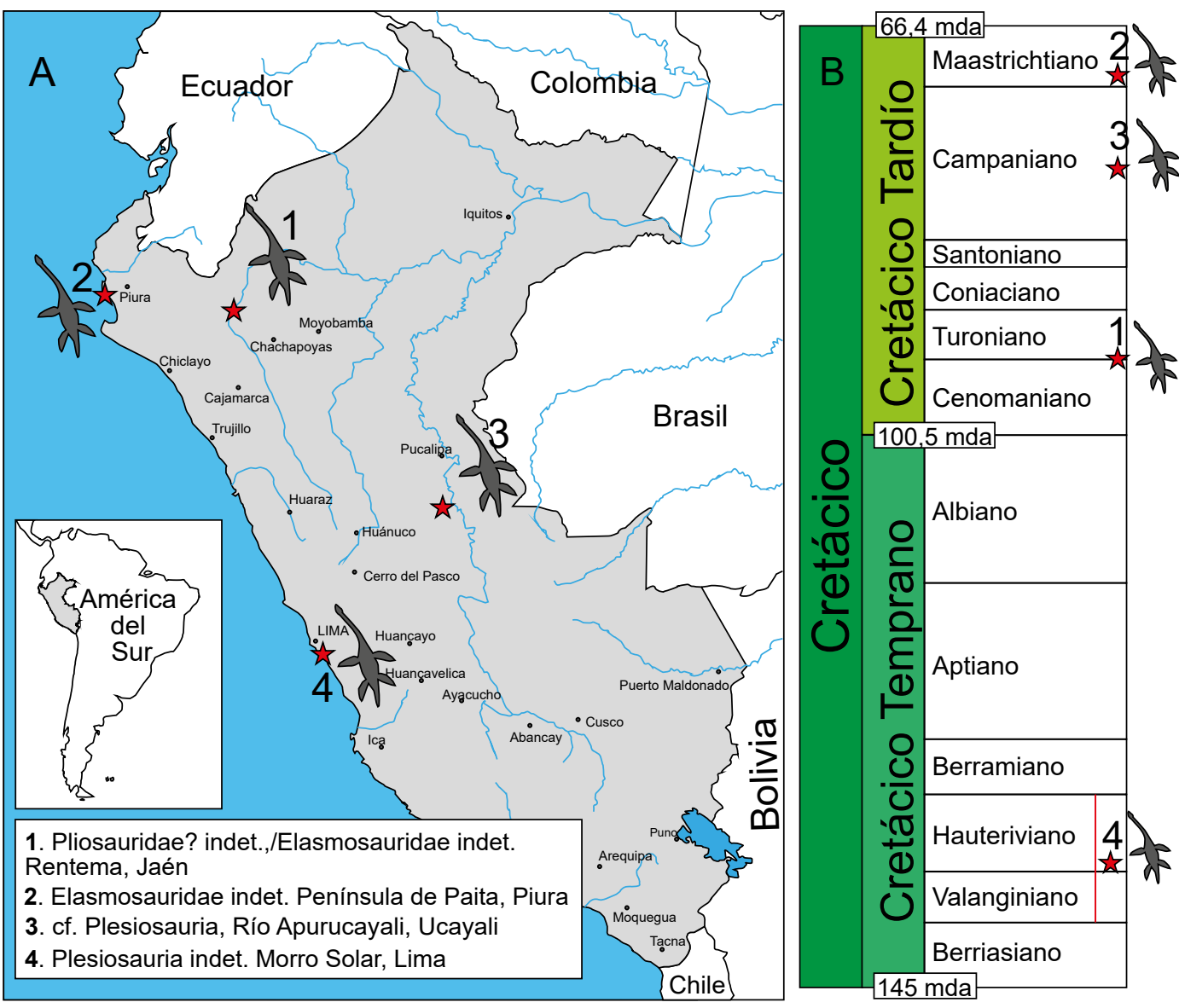

Figura 1. Localidades y posición estratigráfica de hallazgos de plesiosaurios en Perú (datos tomados de Jaillard et al. 1985, Taipe et al. 2004, Poire et al. 2016, Meza-Velez \& O'Gorman 2020). 
Recientemente, Jean-Noël Martinez et al. (2018), mencionaron los fósiles, encontrados 15 años atrás, consistentes en dos piezas diagnósticas identificadas por $\mathrm{N}$. Bardet como un dentario de Elasmosauridae indet. y un basioccipital de Mosasauridae. Martínez et al. (2018) afirmaron que estos fósiles fueron objeto de un estudio preliminar en una tesis de Máster aún no publicada. Estos materiales provienen de Playa Perico, Formación Cenizo, atribuida al Maastrichtiense, aflorante en la península de Paita. Por las características de los fósiles, la localidad, la formación, la edad y la investigadora que los identificó, serían los mencionados por Taipe et al. (2004) y que fueron colectados en 2003.

El objetivo del presente trabajo es hacer un recuento comentado de los registros de plesiosaurios de Perú, describir el material proveniente de la Formación Romirón, Cretácico Superior, Cenomaniano-Turoniano, depositado en el MHN y discutir sus afinidades sistemáticas.

\section{Abreviaciones institucionales}

LACM, Los Ángeles County Museum, California, USA; MHN, Museo de Historia Natural de la Universidad Nacional Mayor de San Marcos; MLP, Museo de La Plata, Argentina; MUSM, Museo de San Marcos; UNMSM, Universidad Nacional Mayor de San Marcos.

\section{Material y métodos}

La presente contribución se sustenta en varias publicaciones y en restos fósiles. Se han utilizado publicaciones científicas (Jaillard et al. 1985, Taipe et al. 2004, Poire et al. 2016, Meza-Vélez \& O’Gorman 2020), así como tesis académicas (Córdova 1986). Los materiales descritos por primera vez en esta revisión pertenecen a las colecciones científicas del Departamento de Paleontología de Vertebrados del Museo de Historia Natural de la UNMSM. En la comparación de proporciones de los centros vertebrales se utilizaron gráficos bivariados considerando los índices propuestos por Welles (1952): $\mathrm{HI}=$ altura de cara articular/longitud del centro vertebral y $\mathrm{BI}=$ medida del ancho del centro vertebral/la longitud del centro vertebral. Los datos de las medidas de los taxones fueron tomados de Welles (1952), O'Gorman et al. (2015b) y O'Gorman (2016).

\section{Resultados y discusión}

A continuación, se brinda un listado completo de los registros de plesiosaurios de Perú en orden estratigráfico con breves comentarios. En el caso del ejemplar MUSM 3894, de una vértebra sacra o caudal proximal y una caudal se brinda una descripción detallada y se discute su posible determinación.

\section{Sistemática Paleontológica}

SAUROPTERYGIA OWEN, 1860

Plesiosauria de BlainVILle, 1835

Plesiosauria indet.

Material.- MUSM 3735 consta de un propodio (hú- mero o fémur), tres vértebras y un ilion (Meza-Vélez \& O'Gorman 2020).

Localidad y horizonte. - Morro Solar, distrito de Chorrillos, Lima, Perú. Formación La Herradura, Miembro La Virgen, Cretácico Inferior, Valanginiano-Hauteriviano (Meza-Vélez \& O'Gorman 2020).

Descripción.- El material MUSM 3735 consiste en una vértebra caudal y vértebras indeterminadas. Asimismo, se preserva parte de un ilium (Meza-Velez \& O'Gorman 2020) y un propodio, siendo imposible determinar si es húmero o fémur.

Comentarios. - MUSM 3735 muestra características que permiten referirlo a Plesiosauria. Las afinidades con dicho orden son manifiestas en el propodio, dada la presencia de forámenes transficiales en el capítulo, su compresión dorsoventral, la presencia de un trocánter dorsal y su expansión distal (Rhodin 1985, Benson \& Druckenmiller 2014). Meza-Vélez y O'Gorman (2020) afirmaron que MUSM 3735 es compatible con la morfología presente en dos familias del orden Plesiosauria: Elasmosauridae y Leptocleididae. Adicionalmente, el propodio de MUSM 3735 presenta contornos similares con el húmero de algunos policotílidos (Meza-Velez \& O'Gorman 2020). En las conclusiones de Meza-Vélez y O'Gorman (2020) se afirmó que el plesiosaurio del Morro Solar es el primer registro de Plesiosauria en el Perú; esta afirmación es acá corregida, dado el anterior registro dado por Jaillard et al. (1985). Sin embargo, sí es el primer registro de Perú correspondiente al Cretácico Inferior.

\section{SAUROPTERYGIA OWEN, 1860 \\ Plesiosauria de BlainVILLe, 1835 \\ Pliosauroidea Seeley, 1874}

\section{Pliosauroidea? indet.}

\section{(Fig. 2)}

Material (según Jaillard et al. 1985). - Consta de cinco vértebras.

Localidad y horizonte. - En la ruta Jaén-Nazareth a $1.5 \mathrm{~km}$ al norte de Rentema, en la cima del Pongo de Rentema, en el grupo Quillquiñan, al norte del Perú. Formación Romirón, Cenomaniano superior (Córdova 1986) o límite Cenomaniano-Turoniano (Jaillard et al. 1985).

Descripción (Jaillard et al. 1985 y comentarios ahí incluidos). - Jaillard et al. (1985) identificaron vértebras cervicales, probablemente de la región anterior del cuello. Dichos autores comentaron que el centro vertebral es corto y sus caras, anterior y posterior son casi planas, dando las siguientes medidas: $\mathrm{L}=53 \mathrm{~mm}, \mathrm{l}=79$ $\mathrm{mm} \mathrm{y} \mathrm{H}=63 \mathrm{~mm}$. Para todas ellas, en el trabajo se afirma que las zonas articulares (facetas costales) a ambos lados son masivas, muy prominentes y únicas. En Córdova (1986) se mencionó el mismo registro sin agregar más información. Tanto en Jaillard et al. (1985) como en Córdova (1986) no se proporcionaron fotos o dibujos de las vértebras. 

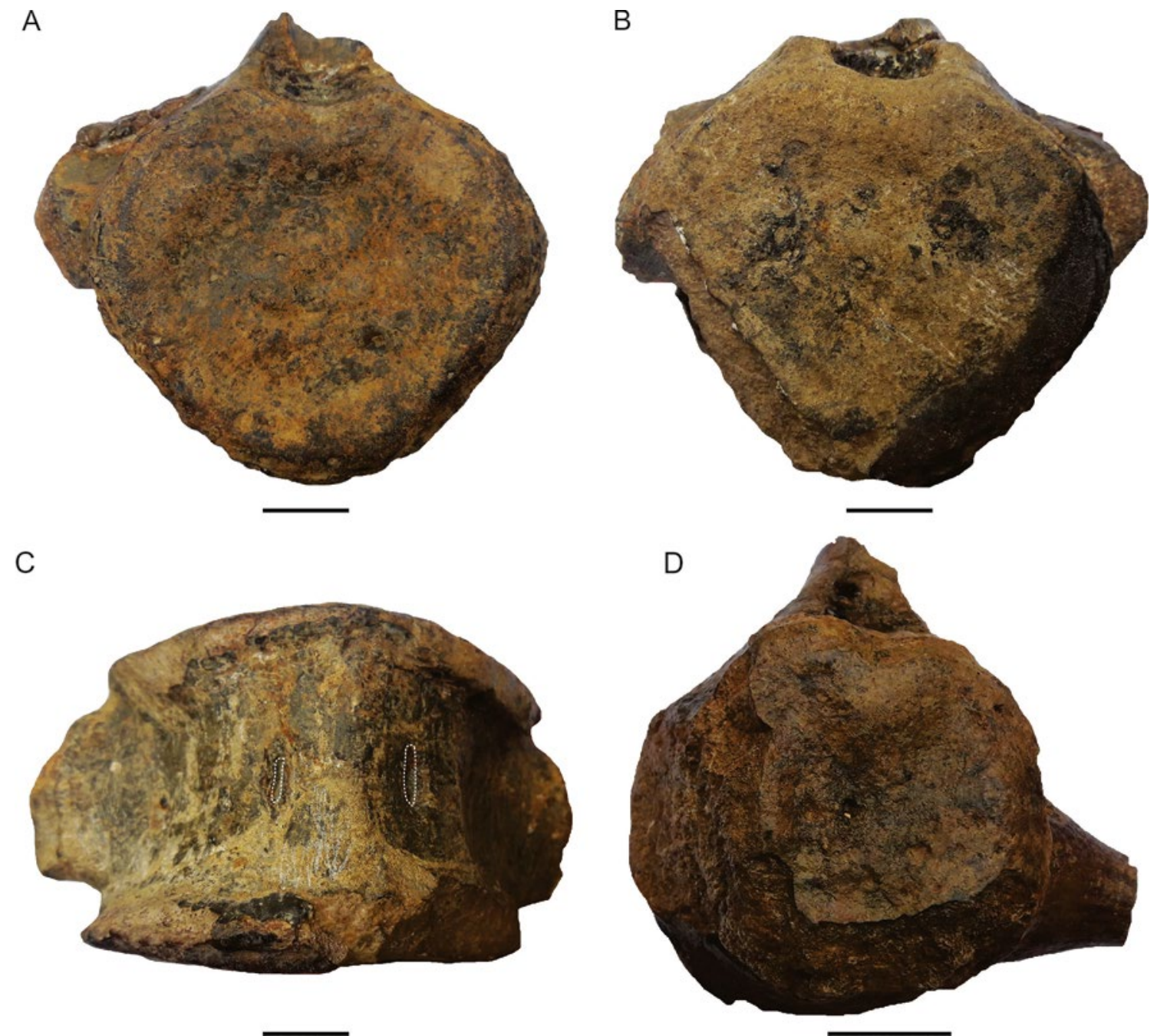

Figura 2. MUSM 3894, Pliosauridae? indet. A-D, vertebra sacra en vista A, posterior; B, anterior; C, ventral, D, vertebra caudal en vista posterior. Escala $=20 \mathrm{~mm}$.

Basándose en la descripción, Jaillard et al. (1985) establecieron que las vértebras descritas eran de la familia Elasmosauridae, considerando que podrían pertenecer a la parte anterior de la región cervical. Con respecto a los materiales, Jaillard et al. (1985:1430) indicaron que una vértebra de dicho material fue llevada a París para su estudio mientras que el resto quedó depositado en el Museo Javier Prado de Lima (actual Museo de Historia Natural de la UNMSM). Sin embargo, revisando la colección del MUSM, solamente se encontraron dos vértebras ingresadas bajo el número MUSM 3894. Dicho material tiene como localidad de origen el Pongo de Rentema, al norte del Perú, Formación Romirón. Pero, la descripción de Jaillard et al. (1985) no permite afirmar que se trate de los mismos materiales y asimismo las determinaciones (Elasmosauridae indet. y Pliosauridae? indet., ver más abajo) tampoco son coincidentes por lo que, nuestra discusión sobre el material MUSM 3894 se basa en las dos vértebras restantes en tanto el material llevado a Francia pueda ser revisado.

Material. - MUSM 3894, una vértebra sacra o caudal proximal y una caudal.

Localidad y horizonte. - El Pongo de Rentema, nor- te del Perú. Formación Romirón, Cenomaniano Superior (Córdova 1986) o límite Cenomaniano-Turoniano (Jaillard et al. 1985).

\section{Descripción. -}

Vertebra sacra. El cuerpo vertebral es más ancho $(82$ $\mathrm{mm})$ que alto $(72 \mathrm{~mm})$ y que largo $(53 \mathrm{~mm})$. Se identifica como vértebra sacra o caudal proximal. El arco neural está fusionado al centro vertebral. Las caras articulares muestran el margen ventral fuertemente convexo y el dorsal bilobulado, formando caras articulares del centro vertebral con forma de corazón o subtriangular ligeramente anficélicas. El proceso transverso es robusto y está desplazado hacia el arco neural, por lo que la costilla articula en una faceta amplia que abarca el cuerpo vertebral y el arco neural, característica de una vértebra sacra de Plesiosauria (Brown 1981). La superficie ventral no evidencia facetas hemales, pero muestra dos forámenes ventrales alargados anteroposteriormente localizados a ambos lados de la línea media, distantes entre sí en $18 \mathrm{~mm}$.

Vertebra caudal. La vértebra caudal está parcialmente conservada e incompleta, con ausencia de una parapófisis debido a rotura; el arco neural se encuentra erosionado e incompleto, faltándole la mayor parte de la espina neural, 
sin embargo, conserva el canal neural. El cuerpo vertebral es más ancho (60 $\mathrm{mm})$ que alto $(55 \mathrm{~mm})$ y que largo (45 $\mathrm{mm}$ ). Las caras articulares son anficélicas, la parapófisis preservada se encuentra proyectada lateralmente hacia el centro del cuerpo vertebral, lo que la caracteriza como una vértebra caudal, y está comprimida dorsoventralmente; además, se aprecian dos forámenes ventrales cercanos entre sí y erosionados. Asimismo, en vista ventral se observan facetas hemales en el margen anterior.

Comentarios. - La vértebra descrita por Jaillard et al. (1985) con sus respectivas mediciones no es ninguna de las actualmente depositadas en el Museo de Historia Natural de la UNMSM. Jaillard et al. (1985) indicó que las facetas costales de dicho espécimen son masivas, prominentes y únicas para todas las vértebras, información que es muy general para poder asignar al espécimen a algún clado dentro de Plesiosauria (entre ellos, Elasmosauridae, como proponen Jaillard et al. (1985)). Asimismo, las medidas aportadas por Jaillard et al. (1985) indican un cuerpo vertebral más alto que largo, proporciones que no coinciden con lo usualmente observado en la parte anterior o media del cuello de los elasmosáuridos (Welles 1952, O'Keefe \& Hiller 2006, Kubo et al. 2012, O'Gorman et al. 2015b, O'Gorman 2016). Por tanto, sobre la base de la descripción de MUSM 3894, no es posible afirmar que el material sea referible a Elasmosauridae, hasta que el material depositado en Francia sea hallado y revisado.

Con respecto a las vértebras MUSM 3894, la comparación de las proporciones de los cuerpos vertebrales con aquellos de elasmosáuridos arroja nueva información. La comparación mediante gráfico bivariado para la vértebra caudal arroja una posición en el límite del morfoespacio ocupado por las vértebras caudales de los elasmosáuridos considerados (Fig. 3).

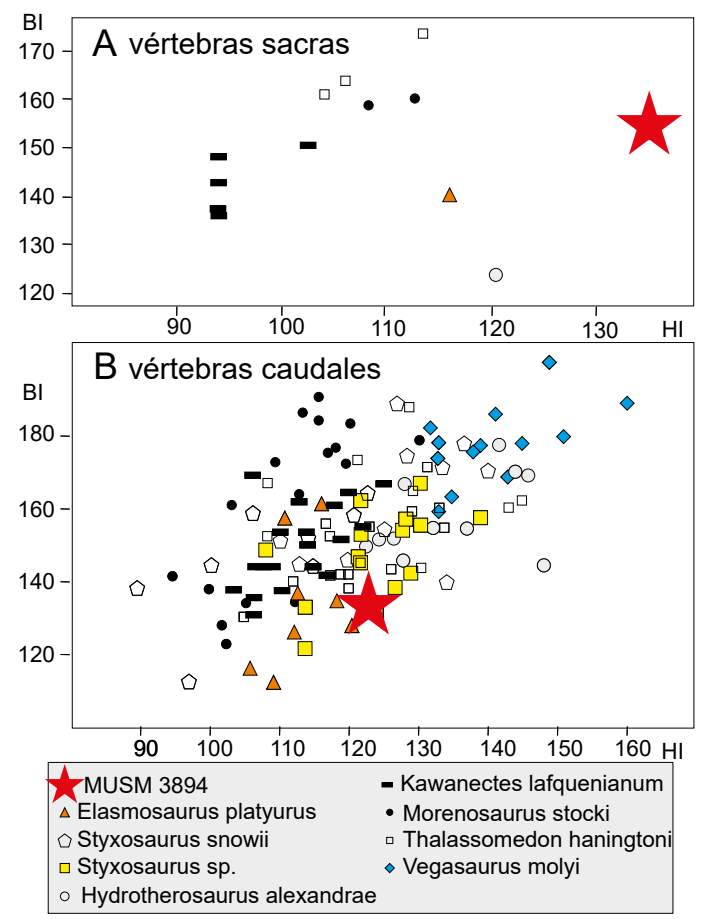

Figura 3. Gráfico bivariado $\mathrm{BI}, \mathrm{HI}$ de vertebras sacras y caudales de elasmosáuridos. (datos tomados de Welles 1952, O'Gorman et al. 2015, O'Gorman 2016).
Por otro lado, la vértebra sacra muestra unas proporciones diferentes a las observadas en otros elasmosáuridos. Por tanto, las proporciones del centro vertebral sacro de MUSM 3894 no son compatibles con las observadas en otros elasmosáuridos. Asimismo, la forma de la cara vertebral de la vértebra sacra se diferencia de lo observado en otros elasmosáuridos y se asemeja a lo observado en algunos pliosáuridos. Así, la forma del cuerpo vertebral de la sacra recuerda a las observadas en algunos pliosaurios como Marmornectes candrewi (Ketchum \& Benson, 2011: Fig 8P-R) o en las vértebras caudales anteriores del bracauquenino Sachicasaurus vitae (Páramo et al. 2018: Fig. 5E, G), en ambos se aprecian centros vertebrales en forma subtriangular, tal como presenta la vértebra sacra de MUSM 3894. Con estas observaciones, es acá referida como Pliosauroidea? indet. Una afinidad con los bracauqueninos no sería incongruente, dado que este grupo presenta un biocrón que alcanza el Cretácico Temprano (Albrigth et al. 2007, Benson \& Druckenmiller 2014, Angst \& Bardet 2016) (Fig. 4).

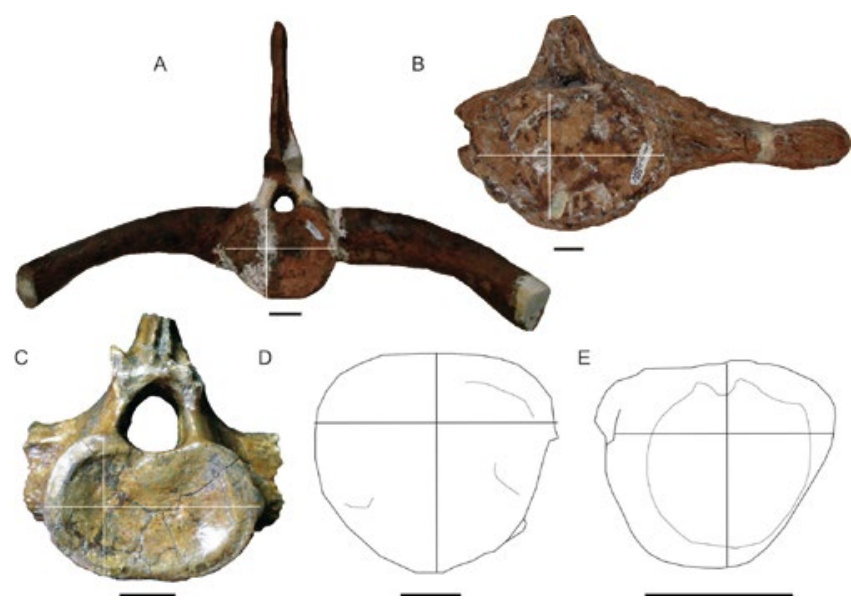

Figura 4. Vértebras sacras de elasmosáuridos, vista articular. A, Thalassomedon haningtoni; B, Elasmosauridae indet. (LACM 2754); C, Kawanectes lafquenianum. D, cara articular de vértebra sacra de Marmornectes candrewi; E, novena centro caudal (D, redibujado de Ketchum y Benson, (2011: fig. 8); E, redibujado de Páramo et al., (2018: fig. 5). Escala $\mathbf{C}=40 \mathrm{~mm} ; \mathbf{B}=20 \mathrm{~mm} ; \mathbf{C}=20 \mathrm{~mm} ; \mathbf{D}=20$ $\mathrm{mm} ; \mathbf{E}=100 \mathrm{~mm}$.

\section{Campaniano}

SAUROPTERYGIA OWEN, 1860

Plesiosauriade BlainVILle, 1835

Plesiosauria indet.

Poiré et al. (2016) registraron un hueso aislado de reptil marino de la Formación Vivian, Cretácico en el río Apurucayali, Ucayali. La edad de la Formación Vivian es de difícil determinación; sin embargo, es posible que pertenezca al Campaniano, según su posición estratigráfica (Vara 2003). El hueso es descrito con morfología subtriangular de $5 \mathrm{~cm}$ de largo y $4 \mathrm{~cm}$ de ancho, asignado tentativamente a un reptil marino (cf. Plesiosauria), (ver comentarios). 


\section{Maastrichtiano}

ELASMOSAURIDAE COPE, 1869

\section{Elasmosauridae indet.}

Taipe et al. (2004) mencionaron el hallazgo realizado por Fischer (1956) de vertebrados marinos, consistente de la mandíbula de un plesiosaurio, acotada en edad entre el Maastrichtiano inferior a la base del Maastrichtiano superior. Esta fue colectada el 2003 (Jean-Noël Martínez et al. 2018) en la Formación Cenizo, Miembro Radiolites Sandstone, playa Perico, localidad La Tortuga, península de Paita, al norte del Perú. Taipe et al. (2004), se refieren a este material como Plesiosauria indet. Posteriormente, Jean-Noël Martínez et al. (2018) se refirieron al mismo material como Elasmosauridae indet., consistente en un dentario identificado por N. Bardet.

Comentarios. - El mencionado material y el estudio de las localidades de donde proviene serán de suma importancia pues permitirá conocer los elementos de la fauna de plesiosaurios del Campaniano-Maastrichtiano de Perú y su relación con otras de la misma edad a lo largo de la costa pacífica de América. Con respecto al material registrado por Poiré et al. (2016: fig. 5), por su morfología es compatible con algún elemento de un miembro de un plesiosaurio. Poiré et al. (2016) muestran una fotografía del material registrado, se observa medianamente conservado, con notorias erosiones en la región distal y está completo, pero una cara está incrustada en el sustrato. El hueso es compatible con un propodio de reptil marino del Mesozoico. Se aprecia un hueso plano hacia la región distal y está fuertemente expandido proporcionalmente a la longitud del propodio, las facetas articulares para los huesos epipodiales forman una $\mathrm{V}$ con una apertura de aproximadamente $135^{\circ}$, formando un margen anterior convexo; la diáfisis es corta y está constreñida centralmente; el capítulo es robusto y presenta una prominencia o proceso. Por las dimensiones y características del fósil, se hace necesario considerar la ontogenia del grupo para hallar afinidad del material con individuos juveniles del orden Plesiosauria. Una segunda opción, pero menos probable, es la afinidad con un propodio de un reptil marino del orden Ichthyosauria, dada la variabilidad de este hueso dentro del grupo (Sander 2000, Maisch 2010).

Conclusiones. - El orden Plesiosuria está registrado en Perú desde el Valanginiano-Hauteriviano hasta el Maastrichtiano. Con respecto a las determinaciones, son en su mayoría referidos como Plesioauria indet., Elasmosauridae indet. o Pliosauridae?, por lo que claramente se requiere un mayor trabajo a futuro. Con respecto al material MUSM 3894, las evidencias aportadas aquí indican que no se trataría de vértebras cervicales ni pertenecientes a la familia Elasmosauridae, sino de dos vértebras, una caudal y una sacra (o caudal proximal) posiblemente de un pliosáurido, y dada la semejanza del centro vertebral subtriangular con la misma estructura de la vértebra caudal del bracauquenino Sachicasaurus vitae, se sugiere afinidad a Brachaucheninae. En cuanto a MUSM 3735, estratigráficamente es el más antiguo y el único del Cretácico Inferior de Perú; mientras que el material registrado por Jaillard et al. (1985) correspondería al primer registro de plesiosaurios en Perú. En resumen, el registro de plesiosaurios de Perú es extenso y abarca casi todo el Cretácico. Sin embargo, la mayoría de los registros consisten hasta ahora en materiales parcialmente diagnósticos, por lo que existe una gran oportunidad de incrementar nuestro conocimiento a futuro.

\section{Literatura citada}

Angst D, Bardet N. 2016. A new record of the pliosaur Brachauchenius lucasi Williston, 1903 (Reptilia: Sauropterygia) of Turonian (Late Cretaceous) age, Morocco. Geological Magazine 153, 449-459. https://doi. org/10.1017/S0016756815000321

Bardet N, Falconnet J, Fischer V, Houssaye A, Jouve S, Pereda Suberbiola X, Pérez-García A, Rage J-C, Vincent P. 2014. Mesozoic marine reptile palaeobiogeography in response to drifting plates. Gondwana Research. 26(3):869-887. https://doi.org/10.1016/j. gr.2014.05.005

Benson RBJ, Druckenmiller PS. 2014. Faunal turnover of marine tetrapods during the Jurassic-Cretaceous transition. Biological Reviews. 89(1):1-23. doi: https://doi. org/10.1111/brv.12038

Brown DS, 1981. The English Upper Jurassic Plesiosauroidea (Reptilia) and review of the phylogeny and classification of the Plesiosauria. Bulletin of the British Museum of Natural History (Geology) 35, 225-234.

Carpenter K. 1999. Revision of North American elasmosaurs from the Cretaceous of the Western Interior. Paludicola 2, 148-173.

Colbert EH. 1949. A new Cretaceous plesiosaur from Venezuela. American Museum Novitates 1420, 1-22.

Córdova A. 1986. Estudio Estratigráfico y Sedimentologico de las Rocas del Cretácico Medio y Superior en el Pongo de Rentema, Río Marañón, Departamento de Amazonas. Tesis para optar el título profesional de ingeniero geólogo. Facultad de Geología, Minas, Metalurgia, Ciencias Geográficas y Mecánica de Fluidos, Universidad Nacional Mayor de San Marcos, Lima, Perú.

Fischer AG. 1956. Cretaceous of Northwest Perú. International Petroleum Co., Ltd. Geological Report WP-13, 74 p. Unpublished. Talara-Perú.

Gasparini Z, De la Fuente M. 2000. Tortugas y plesiosaurios de la formación La Colonia (Cretácico Superior) de Patagonia, Argentina. Revista Española de Paleontología 15, 23-25.

Gasparini Z, O'Gorman J. P. 2014. A new species of Pliosaurus (Sauropterygia, Plesiosauria) from the Upper Jurassic of northwestern Patagonia, Argentina.Ameghiniana 51, 269-283. https://doi.org/10.5710/ AMGH.03.04.2014.2225

Gasparini Z, Spalletti L. 1990. Un Nuevo cocodrilo en los depósitos mareales maastrichtianos de la Patagonia noroccidental. Ameghiniana 27, 141-150.

Hampe 0. 2005. Considerations on a Brachauchenius skeleton (Pliosauroidea) from the lower Paja Formation (late Barremian) of Villa de Leyva area (Colombia). Fossil record 8, 37-51. https://doi.org/10.5194/fr-8-372005 
Jaillard E, Cordova A, Mazin JM, Mourier T. 1985. La transgression du Cénomanien supérieur-Turonien inférieur dans la région de Jaén (Nord du Pérou): données sédimentologiques et stratigraphiques; découverte du premier saurien marin du Pérou. C. R. Acad. Sc. Paris, t. 301, Série II, 20.

Kear BP, Fordyce RE, Hiller N, Siversson M. 2018. A palaeobiogeographical synthesis of Australasian Mesozoic marine tetrapods. Alcheringa: An Australasian Journal of Palaeontology 42, 461-486. https://doi.org/10.1080/ 03115518.2017.1397428

Ketchum HF, Benson RBJ. 2011. A new pliosaurid (Sauropterygia, Plesiosauria) from the Oxford Clay Formation (Middle Jurassic., Callovian) of England: Evidence for a gracile, longirostrine grade of Early-Middle Jurassic pliosaurids. Special Papers in Palaeontology 86, 109-129.

Kubo T, Mitchell MT, Henderson DM. 2012. Albertonectes vanderveldei, a new elasmosaur (Reptilia, Sauropterygia) from the Upper Cretaceous of Alberta. Journal of Vertebrate Paleontology 32, 557-572. https://doi.org/10. $1080 / 02724634.2012 .658124$

Lazo DG, Cichowolski M. 2003. First Plesiosaur remains from the lower Cretaceous of the Neuquén Basin, Argentina. Journal of Paleontology 77: 784-789. https://doi. org/10.1017/S0022336000044498

Martínez JN, Daillie S, Mejía LF, Valdivia LA, Jaillard E, Bardet N. 2018. Vertebrados maastrichtienses de Paita (Perú): primer registro de reptiles marinos mesozoicos en la costa norperuana. Memorias del VI Congreso Latinoamericano de Paleontología de Vertebrados, Volumen 1, Agosto 20 al 25 de 2018, Villa de Leyva, Colombia. ISSN: 2619-2616.

Meza-Velez I, O'Gorman J. 2020. First Plesiosaurian record (Diapsida; Sauropterygia) from the La Herradura formation, (Valanginiane-Hauterivian), Morro Solar, Peru. Cretaceous Research 106: 104-247. https://doi. org/10.1016/j.cretres.2019.104247

O'Keefe FR. 2001. A cladistic analysis and taxonomic revision of the Plesiosauria (Reptilia: Sauropterygia). Acta Zoologica Fennica 213:1-63.

O'Keefe FR, Hiller N. 2006. Morphologic and ontogenetic patterns in elasmosaur neck length, with comments on the taxonomic utility of neck length variables. Paludicola 5:206-229.

O'Keefe FR. 2002. The evolution of plesiosaur and pliosaur morphotypes in the Plesiosauria (Reptilia: Sauropterygia). Paleobiology 28 (1), 101-112. https://doi. org/10.1666/0094-8373(2002)028<0101:TEOPAP> 2.0.CO;2

O'Gorman JP, Lazo DG, Luci L, Cataldo CS, Schwarz E, Lescano M, Aguirre-Urreta MB. 2015a. New plesiosaur records from the Lower Cretaceous of the Neuquén Basin, west-central Argentina, with an updated picture of occurrences and facies relationships. Cretaceous Research 56: 372-387. https://doi.org/10.1016/j.cretres.2015.04.004

O'Gorman JP, Salgado L, Olivero EB, Marenssi SA. 2015. Vegasaurus molyi, gen. et sp. nov. (Plesiosauria, Elasmosauridae), from the Cape Lamb Member (lower maastrichtian) of the Snow Hill Island Formation, Vega Island, Antarctica, and remarks on Wedellian Elasmosauridae. Journal of Vertebrate Paleontology. 35(3):e931285. https://doi.org/10.1080/02724634. 2014.931285
O'Gorman JP, Santillana S, Otero R, Reguero M. 2019. A giant elasmosaurid (Sauropterygia; Plesiosauria) from Antarctica: New information on elasmosaurid body size diversity and aristonectine evolutionary scenarios. Cretaceous Research102, 37-58. https://doi. org/10.1016/j.cretres.2019.05.004

O'Gorman JP. 2012. The oldest elasmosaurs (Sauropterygia, Plesiosauria) from Antarctica, Santa Marta Formation (upper Coniacian? Santonian-upper Campanian) and Snow Hill Island Formation (upper Campanian-lower Maastrichtian), James Ross Island. Polar Research. https://doi.org/10.3402/polar.v31i0.11090

O'Gorman JP. 2016. A small body sized non-aristonectine elasmosaurid (Sauropterygia, Plesiosauria) from the late Cretaceous of Patagonia with comments on the relationships of the Patagonian and Antarctic elasmosaurids. Ameghiniana 53: 245-268. https://doi. org/10.5710/AMGH.29.11.2015.2928

O'Keefe, F.R. 2004. Preliminary description and phylogenetic position of a new plesiosaur (Reptilia: Sauropterygia) from the Toarcian of Holzmaden, Germany. Journal of Paleontology 78, 973-988. https://doi. org/10.1666/0022-3360(2004)078<0973:PDAPPO> 2.0.CO;2

Otero R, Soto-Acuña S, Rubilar-Rogers D. 2015. El registro fósil de plesiosaurios (Sauropterygia) en Chile. Publicación Ocasional del Museo Nacional de Historia Natural, Chile, 63, pp. 151-188, 2015.

Otero R, Soto-Acuña S, Vargas A, Rubilar-Rogers D. 2014. A new postcranial skeleton of an elasmosaurid plesiosaur from the Upper Cretaceous of central Chile and reassessment of Cimoliasaurus andium Deecke. Cretaceous Research50 (2014), 318-331. https://doi. org/10.1016/j.cretres.2014.05.008

Páramo-Fonseca ME, Benavides-Cabra C, Gutiérrez I. 2018. A new large Pliosaurid from the Barremian (Lower Cretaceous) of Sáchica, Boyacá, Colombia. Earth Sciences Research Journal Vol. 22, No. 4 (December, 2018): 223 238. https://doi.org/10.15446/esrj.v22n4.69916

Poiré, D., Iribarne, M., Allcca, M. \& Pérez, L. 2016. Facies sedimentarias, trazas fósiles y restos de vertebrados marinos en sedimentitas estuáricas de la Formación Vivian, Rio Apurucayali, Perú. XVIII Congreso Peruano de Geología (2016).

Rhodin AG. 1985. Comparative chondro-osseous development and growth of marine turtles. Copeia 752-771. https://doi.org/10.2307/1444768

Rieppel O. 1997. Sauropterygia. Introduction, in Callaway, J.M., Nicholls, E.L. (eds.), Ancient marine: San Diego, Academic Press, 107-120.

Rieppel O. 2000. Sauropterygia I. Handbuch der Paläoherpetologie 12A. Verlag Dr. Friedrich Pfeil, Munchen, 134 pp.

Romer AS. 1966. Vertebrate paleontology. 3rd ed. 468 pp. Chicago.

Taipe E, Jaillard E, Jacay J. 2004. Estratigrafía y evolución sedimentológica de la serie del Cretáceo Superior de la península de Paita. Boletín de la Sociedad Geológica del Perú, v. 97 (2004).

Vilas-Bôas I, Carvalho IS. 2001. Répteis marinhos (Mosasauria e Plesiosauria) do Cretáceo Superior da Bacia de São Luís (Maranhão, Brasil). O Cretáceo na Bacia de São Luís-Grajaú, 223-233.

Welles SP. 1952. A review of the North American Cretaceous elasmosaurs. University of California Publications in Geological Sciences 29: 47-144. 
Welles SP. 1962. A new species of Elasmosaur from the Aptian of Colombia and are view of the Cretaceous plesiosaurs. University of California, pp. 1-96. Publications in Geological Sciences 44.

Welles SP, 1943. Elasmosaurid plesiosaurs with description of new material from California and Colorado. Memoirs of the University of California 13,125-254.

Wintrich T, Hayashi S, Houssaye A, Nakajima Y, Sander PM. 2017. A Triassic plesiosaurian skeleton and bone histology inform on evolution of a unique body plan. Science Advances3: e1701144. https://doi.org/10.1126/ sciadv.1701144

Woodward AS. 1891.XLI. Evidence of the occurrence of Pterosaurians and Plesiosaurians in the Cretaceous of Brazil, discovered by Joseph Mawson, Esq., F.G.S. Journal of Natural History 68: 314-317. https://doi. org/10.5962/bhl.title.155081

\section{Agradecimientos / Acknowledgments:}

Nuestro agradecimiento a Alí Altamirano Sierra, investigador del Departamento de Paleontología de Vertebrados del Museo de Historia Natural de la UNMSM, por su colaboración en la prueba de galera.

\section{Conflicto de intereses / Competing interests:}

Los autores no incurren en conflictos de intereses.

\section{Rol de los autores / Authors Roles:}

IMV: Conceptualización; Investigación; Redacción: revisión y edición. JOG: Redacción: revisión y edición.

\section{Fuentes de financiamiento / Funding:}

Esta investigación no recibió ninguna subvención específica de ninguna agencia de financiación, ni del sector comercial o sin fines de lucro.

\section{Aspectos éticos / legales; Ethics / legals:}

Los autores declaran no haber incurrido en aspectos antiéticos en esta investigación. Contamos con la autorización y facilidades del Departamento de Paleontología de Vertebrados del Museo de Historia Natural de la UNMSM. 